\title{
Un contributo allo studio dell'influsso italoromanzo sul dialetto stocavo di Dubrovnik/Ragusa
}

\author{
István Vig \\ Wuppertal \\ vigistvan@yahoo.com
}

Il presente articolo studia un campo di ricerca finora poco praticato dagli studi di contatti linguistici italo-croati, quello dei calchi croati sorti su modelli italoromanzi. Il testo spogliato è la Vita della beata Rosanna, un testo tradotto dall'italiano e sfuggito, insieme ad altri testi contenuti in un codice, all'attenzione degli studiosi della storia letteraria croata. Vengono individuati sette tipi di calchi, tra cui uno che non è stato ancora identificato dalla letteratura specifica.

Parole chiave: contatti linguistici, influsso italoromanzo sul croato, storia della lingua croata, storia della civiltà croata.

\section{Introduzione}

L'influsso di una lingua su un'altra si manifesta in due modi: a livello dei prestiti e a livello dei calchi. Nel primo caso vengono trasferite parole da una lingua all'altra con il conseguente adattamento delle stesse, in modo completo o parziale, al sistema fonomorfologico della lingua mutuante. Nel secondo caso vengono invece coniate espressioni nuove che costituiscono diversi tipi di traduzione delle espressioni presenti nella lingua modello, vengono trasferiti significati nuovi degli elementi lessicali della lingua modello a quelli della lingua mutuante, nonché vengono trasferite funzioni sintattiche degli elementi da una lingua all'altra. I fenomeni del secondo caso sono chiamati calchi.

Per quanto riguarda i contatti italoromanzo-croati l'attenzione degli studiosi si è concentrata finora prevalentemente sullo studio dei prestiti italoromanzi nella lingua letteraria e nei dialetti croati, mentre lo studio dei calchi su modelli italoromanzi nel croato (lingua letteraria e dialetti) ha finora suscitato scarso interesse presso gli studiosi. Con il presente intervento cercheremo di colmare tale lacuna con l'analisi della Vita della beata Rosanna (Život blažene Rosane), un testo in prosa tradotto dall'italiano e tramandato in una copia del XVII secolo (Bratulić 1996: 198). La Vita venne pubblicata con altri testi nel 1913 da Josip Karásek nel volume sotto il titolo Dubrovačke legende ('Leggende di Ragusa') (DL: 1996). 
Karásek ha già sottolineato l'importanza dei testi delle Dubrovačke legende per la storia del dialetto stocavo di Dubrovnik/Ragusa (DL: VII, XXXVIII). Ma i testi della raccolta hanno un'importanza notevole anche per la storia del registro letterario del dialetto croato raguseo.

In questo intervento ci concentreremo come già detto, soltanto sull'analisi del testo della Vita di Rosanna che, oltre ad alcuni passi di contenuto religiosospirituale, appare adatto, per la presenza di ampie parti narrative relative alla vita quotidiana, ad essere confrontato con altri testi di tematica secolare. Infatti, i tipi di calchi della Vita vengono messi a confronto con quelli dei drammi del Rinascimento croato e, in mancanza di uno studio aprrofondito, soltanto con tre drammi di Molière, (L'avaro, Il matrimonio forzato, George Dandin o il marito confuso), tradotti e rimaneggiati in croato nel secolo XVIII a Dubrovnik/Ragusa. Così i confronti contribuiscono anche a stabilire lo spessore e la stabilità dell'influsso italoromanzo sul registro letterario del dialetto croato raguseo sotto un aspetto diacronico più ampio.

\section{Calchi}

Per la classificazione dei calchi ci siamo avvalsi di quella utilizzata dallo slavista tedesco Kurt Schumann (1965).

1. Calco strutturale (Lehnübersetzung). È la traduzione di tutti gli elementi che compongono una parola o un'espressione (Schumann 1965: 62 sgg.).

Od strane + genitivo : da parte di qcn

„...moli ženu da bi od nje strane Elementa brata nje vele srčano pozdravila... " ('pregò la donna di salutare molto cordialmente da parte sua Elemento, suo fratello'; DL: 34; 108).

Od strane + genitivo è attestato nei drammi del Rinascimento croato con numerosi esempi (Benetović, Držić, Gučetić-Bendevišević, Najlešković; Vig 2007: 62).

L'espressione da parte di è attestata in italiano dal sec. XIII in poi, mentre il suo calco croato appare per la prima volta nella cosiddetta Legge di Vinodol (Zakon vinodolski) del 1288 (GDLI 12: 653; AR 16: 654; Vig 2007: 62).

2. Calco semantico (Lehnbedeutung). È l'arricchimento semantico di una parola in seguito al trasferimento di un significato nuovo, da una parola della lingua modello a quella della lingua mutuante (Schumann 1965: 67). Tra i tipi del calco semantico è attestato il

Calco semantico sinonimico (synonyme Lehnbedeutung). In questo caso la base del trasferimento del significato è costituita da un significato comune tra la parola della lingua modello e quella della lingua mutuante (Schumann 1965: 67-68).

Kako stojiš? : come stai?

„...ma recimi kako ti stojiš..." ('ma dimmi, come stai?'; DL: 33; 117).

L'espressione, attestata come hapax (presso Držić) nella lingua dei drammi del Rinascimento croato (Vig 2007: 65), ricorre anche nella traduzione-rifacimento 
di Molière: „...ti nemaš uzroka za tužit se, i zna svak da stojiš dobro.” ('non hai ragione per lamentarti, tutti sanno che stai bene.' (Dubrovačke 2: 97).

La base del trasferimento è costituita dalla coincidenza semantica dell'it. stare e del cr. stajati 'essere, trovarsi' (cfr. stare in punta di piedi: stajati na prstima od nogu; come sta la questione/come stanno le cose?: kako stoji stvar?). Sulla base di queste equivalenze semantiche è stato effettuato il trasferimento del significato 'trovarsi in una determinata condizione, con riferimento allo stato di vita o di salute' al verbo croato. È da osservare che l'equivalente croato dell'it. come stai? è kako si? (lett. 'come sei?').

3. Calco della funzione sintattica di una parola (syntaktische Lehnfunktion eines Wortes). In questo caso su una parola della lingua mutuante viene trasferita una nuova funzione sintattica di una parola della lingua modello (Schumann 1965: 76).

Činiti + infinito : fare (verbo fattitivo)

„...a poslije čini dozvat sve kapetane od konjika...” ('e dopo fece invitare tutti i capitani di cavalleria'; DL: 28; 99).

Nel testo l'uso di činiti in funzione di verbo fattitivo prevale, rispetto a quello del verbo fattitivo croato dati nello stesso testo: „....da se razumijet da ona imaše govorit s kraljem..." ('fece intendere che aveva da parlare con il re'; DL: 23; 79).

La base del trasferimento della nuova funzione sul verbo croato è costituita dall'equivalenza semantica di 'fare' nei verbi rispettivamente italiano e croato.

L'uso di činiti fattitivo è documentato con numerosi esempi nella lingua dei drammi croati del Rinascimento (Benetović, Držić, Gučetić-Bendevišević, Nalješković, Sasin, Vetranović; Vig 2007: 67) e non è raro neanche nelle traduzionirifacimenti dei drammi di Molière (Dubrovačke 2: 92).

4. Reggenza ricalcata di verbi e sostantivi (syntaktischer Lehngebrauch der Rektionen von Verben und Substantiven). La reggenza di un verbo o di un sostantivo della lingua mutuante viene espansa da una reggenza di un verbo rispettivamente di un sostantivo della lingua modello. Tale fenomeno, che non è trattato da Schumann, si rivela un nuovo tipo di calco, finora non individuato.

Govoriti od + genitivo : parlare di qcn/ qcs

„....i sada govorimo od Elementa...('e adesso parliamo di Elemento'; DL: 27; 93).

Tale reggenza è attestata con numerosi esempi non solo nella lingua dei drammi croati del Rinascimento (Benetović, Držić, Vetranović; Vig 2007: 68), ma anche nelle traduzioni-rifacimenti di Molière (Dubrovačke 2: 67).

5. Costrutto sintattico ricalcato (syntaktische Lehnkonstruktion). È l'imitazione di un costrutto sintattico di una lingua straniera (Schumann 1965: 79-80).

Za + infinitivo : per/a + infinitivo

„....Rosana žuđaše imat dijete i Austero za kontentat ženu svoju mnoge lijeke provaše..." ('Rosanna bramava un bambino e Austero, per contentare sua moglie, provò molte medicine'; DL: 15; 44);

Questo e altri esempi ancora, appartengono alla categoria delle proposizioni finali implicite. Come è noto, in questo caso il soggetto della proposizione 
principale è identico a quello della subordinata che viene introdotta dalla preposizione per, più raramente da $a$. Questa costruzione è originariamente sconosciuta alla lingua croata, in cui si usano congiunzioni e verbi coniugati.

La coincidenza delle preposizioni per e $z a$ in sintagmi che indicano 'fine, scopo' (p. es. armadio per abiti: ormar za odijela, le spese per la scuola: troškovi za školu) ha reso possibile l'impiego di $z a$ anche per introdurre proposizoni finali implicite.

Il costrutto è frequnte nella lingua del teatro del Rinascimento croato (Benetović, Držić, Gučetić-Bendevišević, Nalješković; Vig 2007: 70) e anche nelle traduzioni-rifacimenti di Molière (Dubrovačke 2: 61, 69).

6. Locuzioni ricalcate (Lehnwendungen). La locuzione ricalcata è la traduzione della locuzione di una lingua straniera. Lo stesso Schumann sottolinea la difficoltà della distinzione tra i calchi strutturali (Lehnübersetzungen) e le locuzioni ricalcate, aggiungendo che la distinzione dipende dall'interpretazione della locuzione (Schumann 1965: 80-81). Gli esempi, che lo studioso usa per illustrare i calchi strutturali, non contengono verbi, mentre negli esempi per le locuzioni ricalcate i verbi hanno un ruolo centrale. Perciò annoveremo anche noi tra le locuzioni ricalcate soltanto locuzioni che contengono verbi.

Nositi ljubav + dativo : portare amore a qcn

„...ja te molim za ljubav koju mi nosiš..." ('ti prego per l'amore che mi porti'; DL: $17 ; 53)$.

L'espressione è attestata con abbondanza di esempi nei drammi del Rinascimento croato (Držić, Gučetić-Bendevišević, Nalješković, Vetranović; Vig 2007: 72-73) e si trova anche nelle traduzioni-rifacimenti di Molière (Dubrovačke 2: 92). Il calco croato è documentato tra i secoli XVI e XVIII (AR 8: 236). Per l'origine e la diffusione v. Vig (1997, 2007: 33-36).

7. Locuzioni ricalcate con elemento alloglotto (Lehnwendungen mit Fremdelement). Costituiscono un tipo di locuzione ricalcata in cui un elemento del modello in lingua straniera viene trasferito, senza essere tradotto, nella lingua mutuante (Schumann 1965: 83-84).

(U) činiti festu : fare festa

„...poče se činit veću festu neg se je davno vidjela..." ('e si cominciò a fare una festa più grande di quanto da tempo non si fosse vista'; DL: 33; 118).

La locuzione italiana è attestata dal sec. XIII in poi (GDLI 5: 880-881).

\section{Il confronto dei calchi della Vita con quelli del teatro del Rinascimen- to e nelle traduzioni di Molière}

I calchi nella Vita sono costituiti da calchi lessicali e semantici e da calchi sintattici. Lo stesso vale anche per i calchi nei drammi del Rinascimento croato, con un ordine leggermente diverso dei tipi di calchi rispetto a quelli della Vita.

I tipi di calchi nella Vita e nei drammi del Rinascimento croato (DR) sono i seguenti (tra parentesi il numero totale delle ricorrenze): 
Vita

1. locuzioni ricalcate

2. calchi strutturali

3. locuz. ricalcate con elemento alloglotto

4. calchi semantici

5. reggenza ric. di verbi e sostantivi

6. calchi della funz. sintattica di una parola

7. costrutti sintattici ricalcati
$D R$

locuzioni ricalcate

locuz. ricalcate con

elemento alloglotto

calchi semantici

e sostantivi

calchi della funz. sintat-

tica di una parola

costrutti sintattici

ricalcati

In tuttti e due i tipi di testi predominano i calchi lessicali e semantici con le locuzioni ricalcate al primo posto e coincide anche l'ordine decrescente dei tipi di calchi semantici. La tendenza a coniare calchi semantici e lessicali da parte degli autori si spiega con il fatto che il livello semantico, ma soprattutto quello lessicale di una lingua, costituiscono un sottosistema relativamente aperto.

È un fatto singolare che nel testo della Vita, che abbraccia 22 pagine stampate, si trovino 7 tipi di calchi che coincidono con quelli (su un totale di 11 tipi ) dei drammi del Rinascimento croato che hanno un'estensione più ampia. A questo punto la domanda se anche altre leggende contengano altrettanti tipi di calchi o meno, rimane per il momento senza risposta, in attesa di analisi future.

Non pochi calchi del testo della Vita sono identici a quelli dei drammi del Rinascimento croato e anche a quelli dei tre drammi di Molière, come si vede dall'elenco seguente $(\mathrm{DR}=$ drammi del Rinascimento croato, $\mathrm{M}=$ commedie di Molière):

biti od potrebe: DR

hoće rijet: DR, M

imati potrebu: DR

nositi ljubav:DR, M

poći u dobar čas: DR

čuti od + gen.: DR, M

govoriti od + gen.: DR, M

znati od + gen.: DR

$$
\begin{aligned}
& \text { glas od + gen.: DR } \\
& \text { kako stojiš?: DR, M } \\
& \text { od strane + gen.: DR } \\
& \text { u zao čas: DR } \\
& \text { od + gen.: M } \\
& \text { činiti (fattitivo): DR, M } \\
& \text { za + inf.: DR, M }
\end{aligned}
$$

Il fatto che 14 dei 15 calchi elencati, coniati sì su modelli italiani, siano adoperati nei drammi del Rinascimento croato, che non sono traduzioni dall'italiano, bensì testi autonomi, lascia concludere che tali calchi, sorti su modelli italoromanzi, dovessero essere già radicati nel registro letterario di Dubrovnik/ Ragusa nel secolo XVII. Così riceviamo già una risposta, almeno parziale, alla domanda formulata sopra. 


\section{Conclusioni}

L'analisi di un solo testo delle leggende, che è una copia secentesca di un testo tradotto dall'italiano, ha rivelato determinate coincidenze rispettivamente con i calchi e i tipi di calchi nei drammi del Rinascimento croato e nelle traduzionirifacimenti di alcune commedie di Molière, dimostrandone la vitalità nel registro letterario in prosa del dialetto di Dubrovnik/Ragusa. Ci si aspetta dunque che l'analisi completa, ancora da svolgere di tutte le leggende, potrà arricchire questo quadro.

\section{Bibliografia}

AR = Daničić, Đuro et alii [a cura di], (1880-1976). Rječnik hrvatskoga ili srpskoga jezika, Zagreb: Jugoslavenska akademija znanosti i umjetnosti.

Bratulić, Josip (1996). Dubrovačke legende. Josip Karasek, in: Dubrovačke legende [a cura di Josip Karásek], Zagreb: Dora Krupićeva, pp. 195-199.

DL = Karásek. Josip [a c. di], (1996). Dubrovačke legende, Zagreb: Dora Krupićeva. [Ediz. anastatica di Id. (1913). Ragusäische Legende, Prag: Ed. Leschinger]

Dubrovačke = Deanović, Mirko [a cura di], (1972-1973). Dubrovačke preradbe Molièrovih komedija 1-2, Zagreb: Jugoslavenska akademija znanosti i umjetnosti.

GDLI = Battaglia, Sandro (1961-2002). Grande dizionario della lingua italiana, Torino: UTET.

Schumann, Kurt (1965). Zur Typologie und Gliederung der Lehnprägungen, in: Zeitschrift für Slavische Philologie, 32, pp.61-69.

Vig, István (1997). De porter amur à nositi ljubav, in: Hungaro-slavica: Studia in honorem Stephani Nyomárkay [a cura di Janusz Bańczerowski], Budapest: ELTE Szláv Tanszékcsoportja, 338-340.

Vig, István (2007). Sprachkontakte im Alpen-Adria-Raum, Szombathely: Institut für Romanistik der Hochschule Berzsenyi Dániel. 


\section{Contribution to the study on the influence of italo-romance on the croatian language}

A book with the title of Dubrovačke legende ('Legends from Dubrovnik') (Prague, 1913) contains texts which had been unknown to the history of the Croatian language and literature until recent times. The texts were translated from Italian, partly from the 16th and partly from the 17th century. This article covers the study of a 17th century text titled The Life of Blessed Rosanna. 7 different types of calques can be pointed out in it, which can be divided into two major groups: into the one of semantic and lexical calques and into the one of syntactic calques. In them there can be found one (Syntaktischer Lehngebrauch der Rektionen von Verben und Substantiven) which has not been mentioned in the professional literature before. Some of the calques studied show identicity with the ones recorded in the language of the Croatian Renaissance dramas, as well as the calques in the language of Molière translations and adaptations created in the 18th century.

Key words: language contact, calques, influence of Italo-Romance on Croatian, history of the Croatian language, history of the Croatian culture. 
